\title{
Influence of menstrual phase on ventilatory responses to submaximal exercise
}

\author{
T Oosthuyse (BSc Hons) ${ }^{1}$ \\ A N Bosch (PhD) $)^{2}$ \\ ${ }^{1}$ School of Physiology, University of the Witwatersrand Medical School, Johannesburg \\ ${ }^{2}$ UCT/MRC Research Unit for Exercise Science and Sports Medicine, University of Cape Town
}

\begin{abstract}
Objectives. To determine whether an increase in respiratory drive, due to elevated progesterone and oestrogen concentration during various menstrual phases, persists throughout prolonged submaximal exercise and potentially contributes to fatigue. Furthermore, to determine whether the difference in the ventilatory response to exercise from one menstrual phase to another is correlated to the ovarian hormone concentrations.
\end{abstract}

Design. We compared the change in ventilatory parameters during 90 min exercise at $60 \% \mathrm{VO}_{2 \max }$ between the early follicular (EF) and mid-luteal $(\mathrm{ML})$ phase $(N=9)$ and between the EF and late follicular (LF) phase $(N=5)$ in eumenorrhoeic women.

Main outcome measures. Menstrual phase comparisons and correlations between the change in ventilatory parameters (minute ventilation $\left(V_{E}\right)$, respiratory rate $(R R)$, tidal volume) from the EF to ML or from the EF to LF phase and ovarian hormone concentration.

Results. The difference in RR between EF and ML phases correlated to progesterone concentration in the ML phase $(r=0.7, p=0.04)$. In addition, RR was higher during exercise in the ML compared with EF phase for the full duration of exercise by on average $2.3 \pm 2.1$ breaths/min ( $p<$ 0.05). However, no difference in submaximal $\mathrm{VO}_{2}$ between menstrual phases was evident. No significant difference in exercising $-V_{E}$ was observed between menstrual phases, but the change in $V_{E}$ from $E F$ to $M L$ correlated to oestrogen $(r=0.8, p=0.02)$ and progesterone $(r=0.7, p=0.04)$ concentration in the ML phase.

\section{CORRESPONDENCE:}

\section{A N Bosch}

UCT/MRC Research Unit for Exercise and Sports Medicine University of Cape Town

Sports Science Institute of SA

Boundary Rd

Newlands, Cape Town

Tel: 021-650 4578

Fax: 021-686 7530

E-mail: abosch@sports.uct.ac.za
Conclusions. The change in ventilatory parameters from $\mathrm{EF}$ to $\mathrm{ML}$ phase is related to the ovarian hormone concentrations. Therefore inter-individual variability should be considered in menstrual phase comparative studies. Furthermore, the persistently higher RR noted during exercise in the ML phase did not increase metabolic rate, and is therefore not expected to affect rate of fatigue significantly, even during prolonged exercise.

\section{Introduction}

Progesterone increases resting minute ventilation $\left(V_{E}\right)$ by central neural mechanisms in the luteal phase and oestrogen potentiates this action through induction of progesterone receptors in the hypothalamus. ${ }^{1}$ In addition, progesterone increases chemosensitivity, as noted by an increase in the ventilatory response to hypoxia and hypercapnia during the luteal phase compared with the follicular phase ${ }^{6,11}$ or in men treated with medroxyprogesterone acetate (MPA). However, this is not consistently reported, as a recent study did not find an increase in chemosensitivty in the mid-luteal $(\mathrm{ML})$ phase compared with the early follicular (EF) phase. ${ }^{2}$ Furthermore, an increase in body temperature is also known to elevate $V_{E}$ and thus the elevated body temperature associated with the luteal phase may also contribute to the observed hyperventilation. $^{11}$

The effect of elevated progesterone levels on $V_{E}$ during exercise remains equivocal. A number of studies have observed an increase in the ventilatory equivalent (the ratio of $\mathrm{V}_{\mathrm{E}}$ to $\mathrm{VO}_{2}$ ) or a decrease in the end tidal partial pressure of carbon dioxide during incremental exercise to exhaustion in the luteal phase versus the follicular phase or with MPA supplementation in males versus control condition. $4,6,9,11,12$ However, others have found no difference in $V_{E}$ during maximal $^{3,10}$ or submaximal exercise., ${ }^{2,5,8,9}$ Beidleman et al. viewed a progesterone-induced increase in $V_{E}$ to be $a$ potential benefit for athletes when exercising at altitude by possibly causing an increase in oxygen delivery to muscle. ${ }^{2}$ However, they found no improvements in $\mathrm{VO}_{2 \max }$ or endurance capacity at altitude despite a small increase in oxygen saturation ( $3 \%$ ) in the $\mathrm{ML}$ phase compared with the EF phase. ${ }^{2}$ Others view an increased ventilatory response to hypoxia and hypercapnia or to exercise to be deleterious for athletic performance due to the heightened sensation of 
dyspnoea that could limit exercise performance. ${ }^{11}$ Schoene et al. is the only study to report a difference in exercise performance with a coincident increase in $V_{E}$ in the luteal phase versus follicular phase when they observed a shorter time to exhaustion during a maximal ramp test in the luteal phase in non-athletes only. ${ }^{11}$ However, an elevated $V_{E}$ during exercise in the luteal phase in endurance athletes did not compromise maximal time to exhaustion. ${ }^{11}$ Therefore, the negative effects of an increase in $V_{E}$ during exercise due to progesterone can be overcome with training.

Most menstrual phase comparative studies reporting respiratory variables include exercise sessions shorter than 40 minutes. $^{3-6,10-12}$ It would be interesting to investigate whether respiratory differences persist over a longer period of exercise as a prolonged increase in respiratory rate may contribute to fatigue and thereby hamper endurance performance. Therefore, we aimed to compare respiratory variables during prolonged submaximal exercise between menstrual phases. The main focus was a comparison between the EF and ML phase, as the most pronounced difference would be expected between theses phases where progesterone increases from the lowest level in the EF phase to the highest level in the ML phase. Furthermore, the increase in progesterone in the $\mathrm{ML}$ phase coincides with a luteal phase peak in oestrogen and, as mentioned earlier, oestrogen potentiates the increased respiratory drive associated with progesterone. ${ }^{1}$

However, even higher oestrogen concentrations occur in the pre-ovulatory period (referred to as the late follicular (LF) phase) albeit with low but rising progesterone concentrations, making this an interesting phase to consider. Therefore we included a sub-sample group comparison between the EF and the LF phase. The latter menstrual phase is often overlooked, particularly in the longer duration exercise studies. While Hackney et al. claimed to include the LF phase in such a comparison, they reported an average progesterone concentration of $10 \mathrm{nmol} / \mathrm{l}^{8}$, which exceeds the $5 \mathrm{nmol} / \mathrm{l}$ limit for this menstrual phase and would rather suggest a post-ovulatory period. Williams and Krahenbuhl, who observed a significant increase in ventilation rate during short-duration submaximal exercise only at the peak of the progesterone increase in the luteal phase compared with the EF phase, suggest that the inconsistent findings of menstrual phase differences in $V_{E}$ are due to studies not controlling the menstrual phase timing of testing tightly enough. ${ }^{12}$

Lastly, we intend to investigate whether the change in ventilatory parameters observed from the EF to LF or ML phase can be correlated to the oestrogen and progesterone concentration, or the magnitude of the increase in these hormones from the EF phase to either LF or ML phase or the oestrogen to progesterone ratio. Previous studies have been unable to identify relationships between the ovarian hormone concentration and ventilation, ${ }^{2}$ but only considered absolute values for ventilatory parameters instead of increasing the sensitivity of the analysis by rather considering the change in the parameter from one phase to the next.

\section{Methods}

A total of 10 subjects where included in this study (Table I). All subjects where young, healthy medical students who experienced regular menstrual cycles and where classified as sedentary as none participated in regular exercise training and performed less than 2 hours of coincidental exercise per week. Subjects were all non-smokers and had not taken oral contraceptives for at least 1 year before their participation in this study. The study protocol was granted ethical clearance by the Committee for Research in Human Subjects at the University of the Witwatersrand. All subjects gave written consent to participate in the study after the protocol had been carefully explained.

\section{Preliminary screening}

\section{Maximal aerobic capacity}

A discontinuous $\mathrm{VO}_{2 \max }$ test was performed on a stationary bicycle ergometer (Excalibur 911 900, Lode, Groningen, Netherlands). A discontinuous protocol was chosen as it is well tolerated by sedentary subjects and is reported to produce a $\mathrm{VO}_{2 \max }$ value that is comparable to a continuous protocol. ${ }^{7}$ After a 10-minute warm-up subjects performed 3-minute exercise bouts starting at 100 Watts and increasing in increments of 20 Watts interspersed by recovery periods. During the recovery period subjects were allowed to rest for as long as they felt necessary (10 - 20 minutes) or until their heart rate returned to 100 beats/min. During the 3-minute exercise bout subjects breathed through a one-way valve mouthpiece that allowed them to inhale atmospheric air and all expired air was directed through a 3 I mixing chamber into a metabolic cart system (Oxycon-4, Mijnhardt, Bunnik, Netherlands). The oxygen and carbon dioxide analysers were zeroed using $100 \%$ nitrogen gas and calibrated using commercially available gas mixture of known $\mathrm{O}_{2}$ and $\mathrm{CO}_{2}$ content and room air. The system calculated and produced readings of $\mathrm{VO}_{2}$ and $\mathrm{VCO}_{2}$ from conventional equations every 30 seconds. $\mathrm{VO}_{2 \max }$ was considered to have been attained when $\mathrm{VO}_{2}$ differed by less than $1.5 \mathrm{ml} / \mathrm{kg} / \mathrm{min}$ between successive workloads.

\section{Menstrual cycle characteristics}

Subjects recorded their basal oral temperature each morning immediately after waking using a digital thermometer (Vital sign VS-10 Soar Corporation, Japan) accurate to $0.1^{\circ} \mathrm{C}$, in order to identify a biphasic temperature pattern that often characterises a eumenorrhoeic cycle. The timing of the luteinising hormone (LH) surge that induces ovulation was predicted using a home ovulation test (Clearplan, Unipath Ltd., Bedford, England). Subjects exposed the tester to midurine flow of their first morning urine sample starting on the day corresponding to the total number of days in their menstrual cycle minus 15, and continued each morning until a positive result was recorded. 


\section{Experimental protocol}

Each subject completed two or three sessions of 90 minutes of cycling exercise at $60 \% \mathrm{VO}_{2 \max }$ during different phases of the menstrual cycle. The menstrual phases included: (i) EF: 2 - 8 days after the onset of menses; (ii) LF: 2 days before up to the day of a positive LH surge recording; and (iii) ML: 4 - 10 days after a positive LH surge reading.

All 10 subjects performed the experimental trial once in their EF phase, 9 of the subjects performed a ML phase trial and 6 subjects performed a LF phase trial.

A resting blood sample was taken on the day of each trial for the measurement of serum progesterone and oestrogen concentration (Coat-A-Count, Diagnostic Products Corporation, Los Angeles, CA), thereby confirming the subject's menstrual phase. All samples from each subject were analysed in the same assay and intra-assay coefficient of variation was $5.3 \%$ and $4.7 \%$ for oestrogen and progesterone respectively. The accepted range for oestrogen and progesterone concentrations for each menstrual phase were, respectively, $37-220 \mathrm{pmol} / \mathrm{l}$ and $<3 \mathrm{nmol} / \mathrm{l}$ for the EF phase, $360-1377 \mathrm{pmol} / \mathrm{l}$ and $<5 \mathrm{nmol} / \mathrm{l}$ for the LF phase, and $220-955 \mathrm{pmol} / \mathrm{l}$ and $>10 \mathrm{nmol} / \mathrm{l}$ for the $\mathrm{ML}$ phase for oestrogen and progesterone respectively (Coat-A-Count, Diagnostic Products Corporation, Los Angeles, CA). If the measured ovarian hormone concentration fell outside of these acceptable ranges for a particular trial, that trial was excluded from the data analysis.

Each subject performed all trials at the same time of day and the ordering of the trials was randomised with respect to menstrual phase. All experimental trials could not always be completed in the same menstrual cycle - the trial was then completed over two successive cycles.

For 48 hours before the first trial each subject kept a record of all their meals and was asked to follow the same diet as closely as possible before the subsequent trial. Two hours before arrival at the laboratory subjects ate a packed meal (2 $062 \mathrm{~kJ}$ ) consisting of a $175 \mathrm{ml}$ yoghurt, $175 \mathrm{ml}$ orange juice and $30 \mathrm{~g}$ granola bar, providing in total $74 \mathrm{~g}$ of carbohydrate, $14 \mathrm{~g}$ of fat and $12 \mathrm{~g}$ of protein. Subjects were asked to refrain from caffeinated foods and beverages on the day of the trial.

On arrival at the laboratory, body mass was recorded and subjects rested for approximately 20 minutes while seated before a resting blood sample was taken and respiratory and indirect calorimetry measurements were recorded (Oxycon-4, Mijnhardt, Bunnik, Netherlands) for 4 minutes. Subjects then began cycling at $60 \% \mathrm{VO}_{2 \max }$ on the same bicycle ergometer as used in the $\mathrm{VO}_{2 \max }$ test. Respiratory measurements were recorded at 15-minute intervals for 3 minutes during the 90 minutes of exercise. The respiratory variables measured included: minute ventilation $\left(V_{E}\right)$, tidal volume, respiratory rate (RR), oxygen consumption and metabolic equivalents (METS).

\section{Statistical analysis}

A paired $t$ test was used to identify differences in ventilatory parameters between menstrual phases in the EF/ML group and EF/LF group. Pearson's linear regression analysis was conducted to identify relationships between the change in respiratory variables from the EF phase to LF/ML phase and the logarithm of oestrogen and progesterone concentration in the LF/ML phase or the logarithm of the oestrogen to progesterone $(E / P)$ ratio in the $M L$ phase or the magnitude of the increase in oestrogen from EF to LF/ML phase (E-fold) or the magnitude of increase in progesterone from EF to $M L$ phase (P-fold). Data are presented as mean \pm standard deviation and significance was accepted as $p<0.05$.

\begin{tabular}{lc|}
\hline \multicolumn{2}{l}{ TABLE I. Subject characteristics } \\
\hline \multicolumn{2}{l}{ Mean \pm SD } \\
\hline Sample size & 10 \\
Age (years) & $23.1 \pm 1.9$ \\
Body mass (kg) & $55.6 \pm 7.5$ \\
Height (cm) & $160.8 \pm 6.7$ \\
Length of MC (days) & $27.6 \pm 1.8$ \\
Day of ovulation & $14.2 \pm 2.3$ \\
VO 2 max (ml/kg/min) & $29.4 \pm 2.1$ \\
Submaximal exercise Intensity (\% $\left.\mathrm{VO}_{2 \mathrm{max}}\right)$ & $60.2 \pm 3.0$ \\
Submaximal workload (Watts) & $51.5 \pm 14.2$ \\
SD = standard deviation; $M C=$ menstrual cycle. & \\
\hline
\end{tabular}

TABLE II. Resting ovarian hormone concentration profile for the respective menstrual phases

\begin{tabular}{|c|c|c|}
\hline & EF & LF \\
\hline \multicolumn{3}{|l|}{$N=5$} \\
\hline Oestrogen (pmol/l) & $133.1 \pm 54.4$ & $1191 \pm 810$ \\
\hline Progesterone (nmol/l) & $1.8 \pm 0.4$ & $3.9 \pm 1.1$ \\
\hline \multirow[t]{2}{*}{ E-fold } & & $10.3 \pm 6.9$ \\
\hline & EF & ML \\
\hline \multicolumn{3}{|l|}{$N=9$} \\
\hline Oestrogen (pmol/l) & $137.7 \pm 67.1$ & $641.3 \pm 218$ \\
\hline Progesterone (nmol/l) & $1.8 \pm 0.7 \quad 4$ & $5.5 \pm 20.3$ \\
\hline E/P ratio $(\mathrm{pmol} / \mathrm{nmol})^{*}$ & & $16.13 \pm 7.8$ \\
\hline E-fold & & $5.3 \pm 2.6$ \\
\hline P-fold* & & $25.1 \pm 12.2$ \\
\hline \multicolumn{3}{|c|}{$\begin{array}{l}E F=\text { early follicular; } L F=\text { late follicular; } M L=\text { mid-luteal; } E \text {-fold }=\text { the magnitude } o \\
\text { increase in oestrogen concentration above the EF phase; } P \text {-fold }=\text { the magnitude } o \\
\text { increase in progesterone concentration above the EF phase; E/P ratio = oestrogen to } \\
\text { progesterone concentration ratio. }\end{array}$} \\
\hline
\end{tabular}




\section{Results}

All subjects successfully detected a positive reading on the urine $\mathrm{LH}$ test, indicating that they all experience ovulatory cycles. The serum oestrogen and progesterone concentration measured on the day of each trial confirmed that all subjects were in the correct menstrual phase (Table II), except the LF phase trial of 1 subject, and all data from this trial were therefore excluded.

\section{Comparisons between menstrual phases}

No significant difference was observed in minute ventilation expressed in absolute values ( $1 / \mathrm{min}$ ) (Fig. 1a \& b) or relative to $\mathrm{VO}_{2}$ as ventilatory equivalent (data not shown) between the EF and LF or the EF and ML phases at rest or during any time interval during 90 minutes of submaximal exercise. No significant difference was noted in the resting respiratory rate in the EF and LF or EF and ML phase. Furthermore, no difference was observed in respiratory rate at any time interval during exercise between the EF and LF phase (Fig. 1c), but a significantly higher respiratory rate was recorded during exercise at all time intervals in the ML phase compared with the EF phase (Fig. 1d). Resting tidal volume was unchanged between EF and LF or EF and ML phase and at all time intervals during submaximal exercise between the EF and $M L$ phase (Fig. 1 e-f). However, tidal volume tended to be significantly less in the LF phase compared with the EF phase during submaximal exercise when considering the average over 30 - 90 minutes $(p=0.054)$ and $60-90$ minutes $(p=$ 0.065 ) of the exercise period (Fig. 1e). No significant differences where observed between menstrual phases for METS or absolute $\mathrm{VO}_{2}$ (I/min) at rest or during exercise (Fig. g-h).

\section{Test for correlations between ventilatory parameters and ovarian hormones}

No significant correlation was identified between ventilatory parameters, expressed as absolute values and ovarian hormone concentrations. However, when considering the change in a parameter from the EF phase to the LF and ML phase, significant correlations where identified, but only with the change from the EF to $M L$ phase. That is, the change in minute ventilation from the EF to ML phase correlated positively with the magnitude of increase in oestrogen from EF to $\mathrm{ML}$ (E-fold) (Fig. 2a-c).

Furthermore, the change in respiratory rate from EF to ML also correlated positively with the progesterone concentration in the ML phase (Fig. 3a). However, we observed a negative correlation between the change in respiratory rate from $E F$ to $M L$ phase and the E/P ratio (Fig. $3 b$ ).

\section{Discussion}

The most important finding of this study is that the variability in respiratory drive between menstrual phases during submaximal exercise is related to the individual's oestrogen and progesterone concentration on the day.
The current study observed an increase in respiratory rate during exercise in the $\mathrm{ML}$ phase compared with the EF phase. Most previous studies use only minute ventilation or ventilatory equivalents as a means of comparing respiratory drive during exercise between menstrual phases, ${ }^{2-6,8-12}$ and do not consider the contributing variables, respiratory rate or tidal volume. Furthermore, most previous reports have only evaluated ventilatory drive over short exercise periods ${ }^{3-6,10-12}$ whereas the current study demonstrated that the increase in respiratory rate during exercise in the $\mathrm{ML}$ phase continued to persist throughout a prolonged exercise period. Furthermore, this is the first study to demonstrate a significant relationship between respiratory rate and the circulating progesterone concentration. Unlike previous studies that found no significant correlations between the ventilatory response to exercise and ovarian hormones, ${ }^{2}$ we considered the change in the ventilatory parameter between menstrual phases, and so isolated the influence of the intervention (being menstrual phase), thereby increasing the sensitivity of the analysis. Moreover, the negative correlation observed between the change in respiratory rate and the $\mathrm{E} / \mathrm{P}$ ratio does not contradict the significant positive relationship identified between the change in respiratory rate and progesterone concentration, but in fact supports it. That is, a high E/P ratio could be a result of a high oestrogen concentration but low progesterone concentration and thus the lower progesterone concentration would result in less of a difference in respiratory rate between the EF and the ML phase, whereas a low E/P ratio could be a result of a relatively high progesterone concentration and thus a marked increase in respiratory rate from EF to $\mathrm{ML}$ phase. Nonetheless, the average increase in respiratory rate in the ML phase relative to the EF phase was only 2.3 \pm 2.1 breaths/min (ranging between 0.13 and 5.6 breaths/ min) and this extra demand on respiratory muscles did not significantly increase metabolic demand, as demonstrated by a similar rate of oxygen consumption between the EF and $M L$ phase. We further tested the effect of this change in respiratory rate from $E F$ to $M L$ phase on metabolic rate, by testing for a correlation between the change in oxygen consumption from EF to $\mathrm{ML}$ and the change in respiratory rate or minute ventilation from EF to ML phase and found no significant relationships (data not shown). Therefore we would expect that the slightly greater work demand on the inspiratory muscles in the ML phase would have a negligible effect on rate of fatigue or exercise performance, even during prolonged exercise.

Furthermore, the increase in respiratory rate during exercise in the ML phase of the current study did not appear to have a significant influence on minute ventilation. In contrast, some previous studies have observed greater minute ventilation during exercise in the luteal phase compared with the follicular phase, ${ }^{4,6,9,11,12}$ but this has not been consistently reported. ${ }^{2,3,5,8,10}$ However, despite the finding of no difference in minute ventilation between EF and ML in this current study and no significant correlation between absolute 

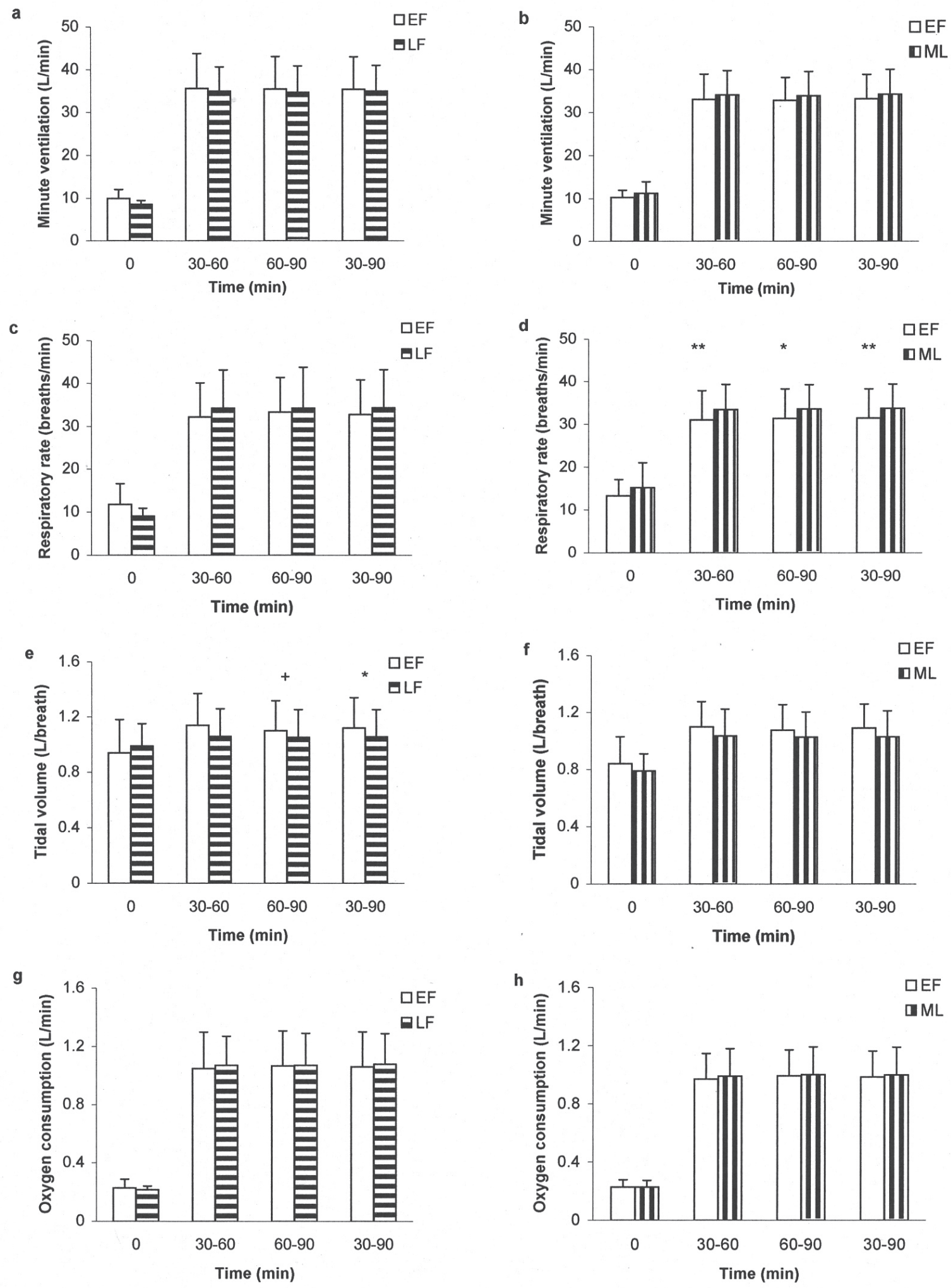

Fig. 1. Comparison of the following ventilatory parameters (mean and standard deviation): minute ventilation (a and $b$ ); respiratory rate ( $c$ and $d$ ); tidal volume (e and $f$ ); and oxygen consumption ( $g$ and $h$ ), at rest and averaged over 30-minute time intervals during 90 minutes of submaximal exercise between either the EF and LF or EF and ML phases. * and ** denotes significance between identified menstrual phases with $\mathrm{p} \leq 0.05$ and $\mathrm{p}<0.02$, respectively. ${ }^{+}$denotes a trend for a difference between identified menstrual phases $\mathrm{p}=0.065$. 


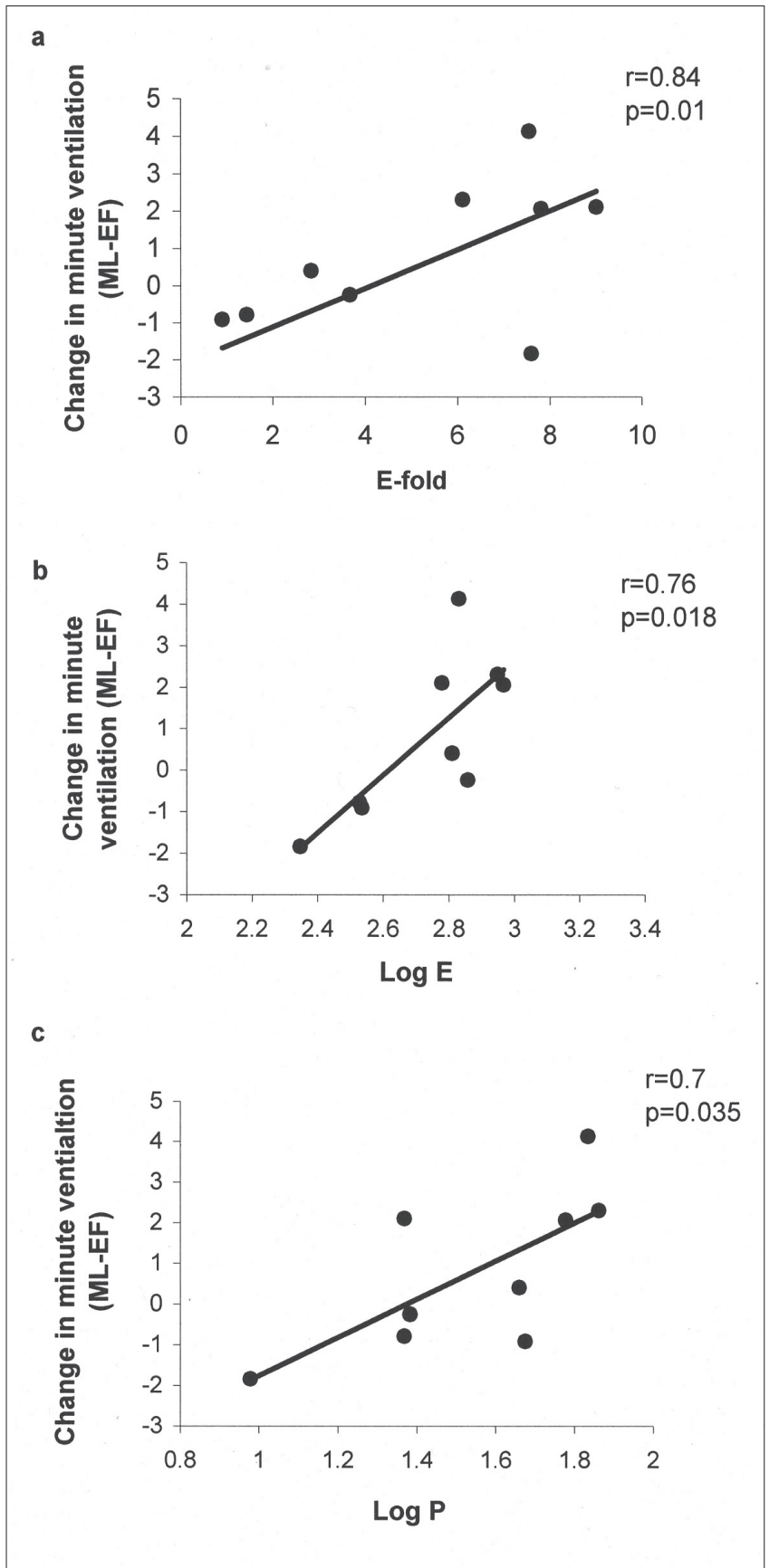

Fig. 2. Significant correlations identified between the change in minute ventilation from the EF to $M L$ phase during submaximal exercise and the magnitude of increase in oestrogen concentration from the EF to $M L$ phase (E-fold) (a); logarithmic function of oestrogen concentration (b); and logarithmic function of progesterone concentration (c) in the ML phase.

minute ventilation and ovarian hormones, we did observe a significant relationship in the change in minute ventilation from $\mathrm{EF}$ to $\mathrm{ML}$ and the oestrogen and progesterone concentration, and the magnitude of increase in oestrogen between these menstrual phases. We therefore suspect that individual variability in the increase in oestrogen and progesterone in the $M L$ phase may explain the inconsistency in the literature
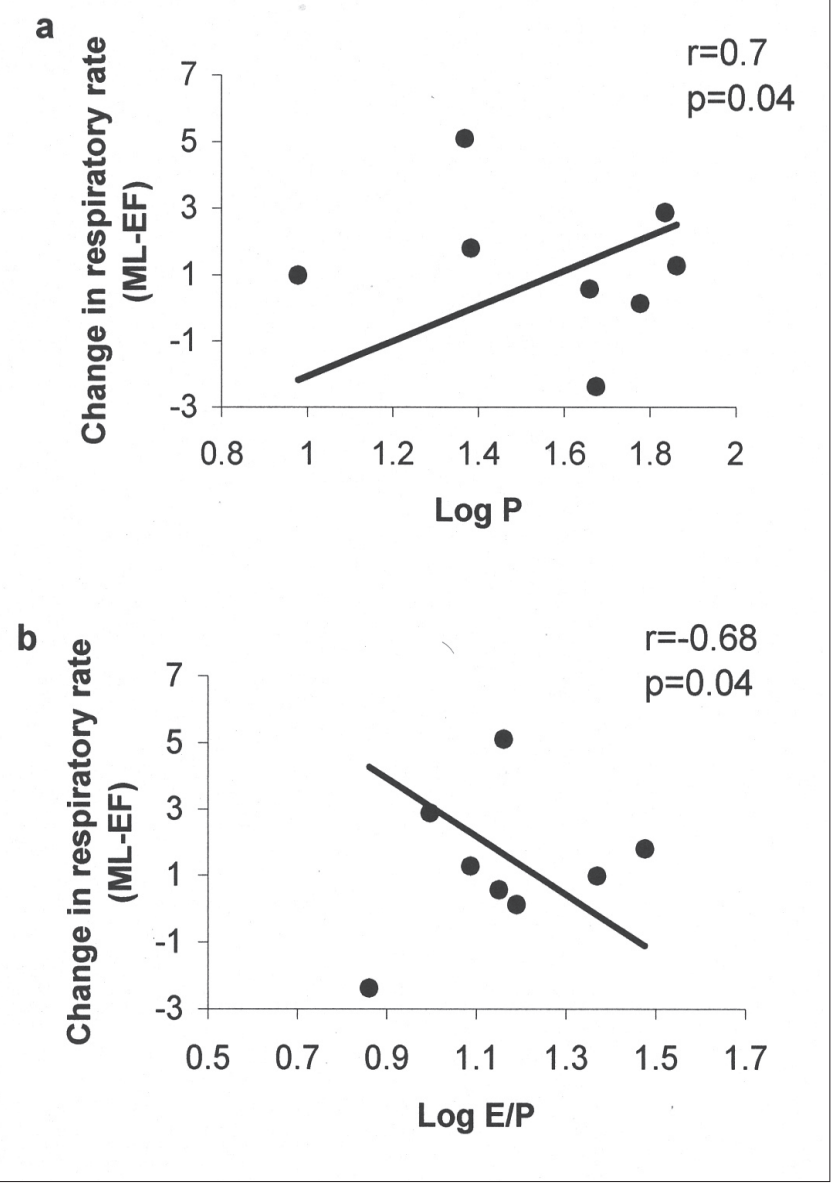

Fig. 3. Significant correlations identified between the change in respiratory rate from the EF to $M L$ phase during submaximal exercise and the logarithmic function of progesterone concentration (a) and the logarithmic function of the oestrogen to progesterone (EIP) ratio (b) in the $M L$ phase.

regarding menstrual phase variation in ventilation during exercise. Furthermore, the significant correlations observed with not only progesterone concentration but also oestrogen and degree of increases in oestrogen are supposedly related to earlier reports describing progesterone's dependence on oestrogen in order to cause the heightened ventilatory response. ${ }^{1}$ The LF phase characterised by the preovulatory surge in oestrogen with no or minor increases in progesterone did not appear to influence respiratory rate or minute ventilation. However, it is possible that the major peak in oestrogen during this pre-ovulatory phase may prime the hypothalamic nuclei cells for progesterone receptor induction and so facilitate the ventilatory response in the luteal phase.

The sample sizes employed in the current study may be considered relatively small and the possibility of incurring a type I error in the interpretation of the significant correlations must be considered. However, the relation identified in the current study between the ventilatory response to exercise and the ovarian hormones is not just based on a single 
significant correlation. Rather, a number of significant correlations were identified, with not just one ventilatory parameter, but with both changes in minute ventilation and respiratory rate from the EF to $M L$ phase when related to not only just (say) progesterone concentration but also oestrogen concentration or E/P or E-fold. Therefore it is unlikely that all of these relations were identified by chance.

The tendency for tidal volume during exercise to be less in the LF phase compared with the EF phase is unexpected and remains unexplained. However, in our small sample group the observed difference in tidal volume did not have a significant effect on minute ventilation. Future studies should investigate respiratory responses to prolonged exercise in the LF phase employing a larger sample size.

The overriding evidence for a greater ventilatory response at rest in the luteal phase versus the follicular phase ${ }^{1,11,12}$ was not evident in the current study. However, the premeasurement resting period (which was not always strictly regulated) and the short recording interval may have been insufficient to establish a true resting reading and so a nonsteady-state condition may have obscured our comparison at rest.

In conclusion, the current study supports reports of greater ventilatory drive in the ML phase compared with the EF phase during submaximal exercise. We noted that the response persists during prolonged exercise but nonetheless the increased demand on respiratory muscle is small and we expect that it should not exacerbate fatigue. Furthermore, the change in ventilatory response during submaximal exercise between the EF and ML phase is related to both the oestrogen and progesterone concentration and thus both of the ovarian hormone concentrations determine the response of an individual in the luteal phase. Therefore inter-individual variability in ovarian hormone concentration must be considered when studying ventilatory responses in women.

\section{Acknowledgements}

This study was funded by the Medical Research Council of South Africa and the University of the Witwatersrand Research Committee.

\section{REFERENCES}

1. Bayliss DA, Millhorn DE. Central neural mechanisms of progesterone action: application to the respiratory system. J Appl Physiol 1992; 73: 393404.

2. Beidleman BA, Rock PB, Muza SR, Fulco CS, Forte VA Jr, Cymerman A. Exercise VE and physical performance at altitude are not affected by menstrual cycle phase. J Appl Physiol 1999; 86: 1519-26.

3. Bemben DA, Salm PC, Salm AJ. Ventilatory and blood lactate responses to maximal treadmill exercise during the menstrual cycle. J Sports Med Phys Fitness 1995; 35: 257-62.

4. Bonekat HW, Dombovy ML, Staats BA. Progesterone-induced changes in exercise performance and ventilatory response. Med Sci Sports Exerc 1987; 19: 118-23.

5. De Souza MJ, Maguire MS, Rubin K, Maresh CM. Effects of menstrual phase and amenorrhea on exercise responses in runners. Med Sci Sports Exerc 1990; 22: 575-80.

6. Dombovy ML, Bonekat HW, Williams TJ, Staats BA. Exercise performance and ventilatory response in the menstrual cycle. Med Sci Sport Exerc 1987; 19: 111-7.

7. Duncan GE, Howley ET, Johnson BN. Applicability of $\mathrm{VO}_{2 \max }$ criteria: discontinuous versus continuous protocols. Med Sci Sports Exerc 1997; 29: 273- 8.

8. Hackney AC, Curley CS, Nicklas BJ. Physiological responses to submaximal exercise at the mid-follicular, ovulatory and mid-luteal phases of the menstrual cycle. Scand J Med Sci Sports 1991; 1: 94-8.

9. Jurkowski JEH, Jones NL, Toews CJ, Sutton JR. Effects of menstrual cycle on blood lactate, $\mathrm{O} 2$ delivery, and performance during exercise. J Appl Physiol 1981; 51: 1439-99.

10. Lebrun CM, McKenzie DC, Prior JC, Taunton JE. Effects of menstrual cycle phase on athletic performance. Med Sci Sports Exerc 1995; 27: 437 44.

11. Schoene RB, Robertson HT, Pierson DJ, Peterson AP. Respiratory drives and exercise in menstrual cycles of athletic and nonathletic women. $J \mathrm{Appl}$ Physiol 1981; 50: 1300-5.

12. Williams TJ, Krahenbuhl GS. Menstrual cycle phase and running economy Med Sci Sports Exerc 1997; 29: 1609-18. 